\title{
Il Fast-Track delle fratture di femore prossimale over 65: analisi dell'epicrisi e sviluppo di modelli organizzativi efficaci
}

\author{
G.M. Calori ${ }^{1}$, M. Colombo ${ }^{1, a}(\bowtie)$, A. Campi ${ }^{2}$, B. Di Maggio ${ }^{3}$, V. Caiaffa ${ }^{4}$, A. Momoli ${ }^{5}$, F. Caroli ${ }^{6}$, G. Longo ${ }^{7}$, \\ F. Donelli ${ }^{8}$, F. Randelli ${ }^{9}$, P. De Simoni ${ }^{10}$, A. Pellegrino ${ }^{11}$, F. Falez ${ }^{12}$, G. Sessa ${ }^{13}$ \\ ${ }^{1}$ U.O.C. di Chirurgia Ortopedica ricostruttiva, revisioni protesiche e sepsi dell'apparato locomotore, ASST \\ Pini-CTO, Università di Milano, Milano, Italia \\ ${ }^{2}$ Divisione di Ortopedia, Azienda Ospedaliera San Giovanni-Addolorata, Roma, Italia \\ ${ }^{3}$ U.O.C. di Ortopedia e Traumatologia, Ospedale di Piedimonte Matese, Piedimonte Matese, Italia \\ ${ }^{4}$ Struttura Complessa Ortopedia e Traumatologia "Di Venere", Bari, Italia \\ ${ }^{5}$ U.O.C. di Ortopedia e Traumatologia, Ospedale S. Bortolo, Vicenza, Italia \\ ${ }^{6}$ U.O.C. di Ortopedia e Traumatologia Romano, ASST Bergamo Ovest, Romano di Lombardia, Italia \\ ${ }^{7}$ U.O.C. di Ortopedia e Traumatologia, Azienda Ospedaliera per l'Emergenza "Cannizzaro", Catania, Italia \\ ${ }^{8}$ Università degli Studi di Milano, Milano, Italia \\ ${ }^{9} 1$ a divisione, ASST Pini-CTO, Università di Milano, Milano, Italia \\ ${ }^{10}$ U.O.C. di Ortopedia e Traumatologia, Azienda Ospedaliera Ospedali Riuniti Marche nord, Pesaro, Italia \\ ${ }^{11}$ U.O.C. di Orto-Traumatologia, Ospedale S.G. Moscati, Aversa, Italia \\ ${ }^{12}$ U.O.C. di Ortopedia, Ospedale Santo Spirito, Roma, Italia \\ ${ }^{13}$ Dipartimento di Chirurgia Generale e Specialistiche II, Sezione di Ortopedia e Traumatologia, Azienda \\ Ospedaliero-Universitaria Policlinico Vittorio Emanuele, Catania, Italia \\ amaz.colombo@hotmail.it
}

\begin{abstract}
FAST-TRACK OF PROXIMAL FEMUR FRACTURES IN PATIENTS OVER 65 YEARS: ANALYSIS OF THE EPICRISIS AND DEVELOPMENT OF EFFECTIVE ORGANIZATIONAL MODELS

During the Trauma Meeting 2017 in Riccione an important Round Table took place entitled "Analysis of the epicrisis and development of effective organizational models" within the session "Fast-track of proximal femur fractures in patients over 65 years". Given the great relevance of the topic and the precious contribution of the participants, it was decided to create the following scientific manuscript of Discussion and Consensus, which contains the answers to the five questions that were the subject of the Round Table.
\end{abstract}

Pubblicato online: 19 febbraio 2018

(c) Società Italiana Ortopedici Traumatologi Ospedalieri d'Italia 2018

\section{Introduzione}

Durante il Trauma Meeting 2017 di Riccione si è tenuta un'importante Tavola Rotonda dal titolo "Analisi dell'epicrisi e sviluppo di modelli organizzativi efficaci" all'interno della sessione "Il Fast-Track delle fratture di femore prossimale over 65”.

Data la grande rilevanza e attualità del tema trattato e dato il prezioso contributo dei partecipanti è stato deciso di creare il seguente lavoro scientifico di Discussione e Consenso al cui interno sono contenute le risposte alle cinque domande che sono state argomento della Tavola Rotonda.

Il Fast-Track, per come è attualmente proposto, rappresenta un modello organizzativo valido alla luce dello stato dell'arte e di fatto vigente in Italia? Testimonianze, esperienze e risultati preliminari Penso che il Fast-Track come proposto possa considerarsi valido in alcune realtà e non in altre. L'Italia ospedaliera e ortopedica presenta tante realtà diverse, troppo diverse.

(A. Campi) 
Ritengo che il Fast-Track sia certamente, allo stato attuale, una procedura valida organizzativamente e chirurgicamente per la cura delle fratture del collo femore nei pazienti over 65 anni. Permangono perplessità, più volte avanzate, sulla necessità di garantire a questi fratturati un percorso prioritario rispetto a pazienti fratturati in altri distretti scheletrici, comunque presenti in ogni reparto di traumatologia, che tendono ovviamente a rinvii maggiori del trattamento chirurgico, specialmente in quelle situazioni lavorative in cui spending review e blocco del turn-over prolungato per il personale medico e paramedico hanno creato inevitabili carenze di personale.

La soglia dell' $80-90 \%$ dei pazienti da operare nelle 48 ore appare purtuttavia, anche in queste difficili situazioni ambientali, raggiungibile, rappresentando essa la stragrande maggioranza dei pazienti da sottoporre a intervento chirurgico che non presentino gravi comorbidità che giustifichino il ritardato trattamento; in particolare, i gravi broncopatici con annesse patologie epatiche e nefrologiche e i cardiopatici in trattamento farmacologico spesso con doppia antiaggregazione nei quali, spesso, l'eccessiva velocità del trattamento può addirittura creare problemi maggiori di una prudente, ponderata e giusta attesa nei tempi.

Questo 10\% rappresenta proprio questi pazienti "difficili" a maggior rischio in cui ritengo sia utile non soltanto una sempre maggiore azione congiunta polispecialistica che permetta, in breve tempo, un inquadramento clinico delle numerose patologie presentate, ma la costituzione di un vero e proprio team dedicato alla cura chirurgica di questi pazienti che riesca, addirittura prima delle 48 ore, a garantire il trattamento chirurgico necessario che spesso si rivela l'unico strumento per evitare complicanze maggiori legate all'allettamento e all'immobilità forzata.

(B. Di Maggio)

Il Fast-Track, nella realtà italiana, segue percorsi diversi; nonostante ciò, serve di stimolo a effettuare interventi negli over 65 in tempi brevi, pur essendo molto lontani dalla media europea.

(V. Caiaffa)

Il Fast-Track è sicuramente un modello organizzativo valido e rappresenta il primo passo verso un miglioramento futuro del percorso del paziente nel processo di cura.

(A. Momoli)

Alla luce della nostra esperienza, la proposta di Fast-Track è applicabile solo in pazienti giovani che utilizzino il servizio di riabilitazione come servizio ambulatoriale. Purtroppo, mancando sul territorio strutture che possano accogliere il paziente anziano, siamo costretti a trasferire i pazienti più longevi presso il servizio di riabilitazione specialistica con ricovero di almeno 2-3 settimane, essendo i famigliari spesso non in grado di gestire queste situazioni. Il tentativo di
Fast-Track proposto prevede, anche a livello anestesiologico, anestesie di breve durata e con terapia analgesica che permetta al paziente di essere mobilizzato nell'immediato postoperatorio. Quello che invece nel Fast-Track è sicuramente applicabile è la gestione perioperatoria del paziente, che risulta essere più snella utilizzando tecniche chirurgiche con vie più anatomiche che permettano la mobilizzazione rapida del paziente anziano.

(F. Caroli)

L'attuale modello di Fast-Track in Italia si basa soprattutto sul cercare di conseguire un risultato (operare questi pazienti entro 48 ore) e meno sui metodi per ottenerlo. Quindi più che un metodo, è un fine. Molto è lasciato alle Regioni, agli Enti e a noi stessi ortopedici senza una vera indicazione, linea guida, comportamentale. Di fatto è un grande dispendio di energie, soprattutto di noi ortopedici, senza un vero risultato eccellente. Certo vi è stato un miglioramento.

(F. Randelli)

Le attuali linee guida raccomandano di effettuare il trattamento chirurgico delle fratture di femore entro le 24-48 ore. Dati pubblicati dall'Agenzia Nazionale per i Servizi Sanitari Regionali (AGENAS) evidenziano che nel periodo 20112016 sono state più di 670.000 le giornate di degenza risparmiate, di cui 200 mila nel 2015 grazie all'applicazione del Fast-Track. La percentuale di interventi entro le 48 ore, che nel 2010 si attestava al 31\%, nel 2015 è passata al 55\%. Per questo indicatore, il regolamento del Ministero della Salute sugli standard quantitativi e qualitativi dell'assistenza ospedaliera ha fissato come valore di riferimento lo standard minimo al 60\%. A livello intra- e interregionale si osserva una notevole variabilità, con valori per struttura ospedaliera che vanno da un minimo di $1 \%$ a un massimo di $97 \%$. Il FastTrack per le fratture del collo del femore rappresenta sicuramente un valido strumento per raggiungere gli obiettivi proposti dal Ministero della Salute. Nella nostra azienda, il Fast-Track prevede che il paziente arrivato al pronto soccorso con sospetto clinico di frattura di femore venga sottoposto a prelievo ematico per emocromo con formula leucocitaria, funzionalità epatica e renale, elettroliti, coagulazione, proteinemia ed emogruppo, radiografia del bacino e anca, del torace ed elettrocardiogramma. Eseguito il ricovero, il medico ortopedico e l'anestesista valutano le condizioni del paziente e provvedono all'eventuale richiesta di approfondimenti diagnostici. Adottando tale percorso, nella nostra azienda siamo riusciti a superare la percentuale del $90 \%$ di pazienti trattati entro le 48 ore.

(P. De Simoni) 


\section{Basandosi su criteri di EBM, cosa è effettivamente prioritario e quali strategie debbono essere adottate per migliorare la qualità e la sicurezza delle cure di tali pazienti?}

Ẽ accertato che è prioritaria la tempistica del trattamento di queste fratture, ma è non secondario tener conto che è altrettanto importante sia lo stato generale del paziente sia la corretta esecuzione del gesto chirurgico. Fare presto è bene, ma se per fare bene è necessario attendere per avere condizioni più favorevoli, allora meglio agire in condizioni ottimali. (A. Campi)

Le EBM dimostrano senza dubbio alcuno il beneficio che un intervento precoce, in questi pazienti, assicura sulla sopravvivenza e sull'outcome dei pazienti affetti da frattura del collo femore.

\section{(B. Di Maggio)}

Un precoce intervento nel paziente stabilizzato o non stabilizzabile.

(V. Caiaffa)

Le fratture del collo del femore sono una patologia frequente nell'età senile a causa dell'osteoporosi. Le Linee Guida internazionali asseriscono che l'attesa per l'intervento comporta un maggiore rischio di mortalità e disabilità per il paziente, pertanto oggigiorno è fondamentale eseguire l'intervento entro le $24-48$ ore dall'ingresso in ospedale. Interventi eseguiti oltre tale periodo determinano un incremento del rischio di complicanze maggiori, quali embolia polmonare, sepsi, insufficienza renale con dialisi, insufficienza respiratoria; inoltre, i trattamenti eseguiti oltre le 24 ore aumentano il rischio di eventi avversi definiti "minori", quali delirium, trombosi venose profonde, infezioni, eventi cardiaci e polmonari senza necessità di trattamenti intensivi. Pertanto, il paziente con sospetta frattura del femore deve essere tempestivamente valutato dal medico del Pronto Soccorso entro 4 ore dall'accesso all'ospedale e successivamente operato nel più breve tempo possibile. Per consentire ciò, è necessaria la formalizzazione di un gruppo aziendale multidisciplinare e multiprofessionale che si confronti periodicamente, e che comprenda l'ortopedico, il geriatra/internista, l'anestesista, il cardiologo, il fisiatra, l'infermiere, il fisioterapista, l'assistente sociale.

$\mathrm{Al}$ momento del ricovero è opportuno effettuare una valutazione e gestione di multimorbilità e dolore, stato cognitivo, tono dell'umore, stato nutrizionale, stato funzionale pre-frattura, nonché condizioni sociali ed economiche. Inoltre, è indispensabile attuare una profilassi antitrombotica, gestire la terapia antiaggregante e anticoagulante praticata al domicilio, effettuare un'opportuna profilassi antibiotica preoperatoria.

Un intervento precoce richiede la disponibilità di sale operatorie, staff chirurgico e anestesiologico al fine di minimizza- re il ritardo dell'intervento per ragioni non cliniche. Per consentire ciò sono necessari percorsi separati fra traumatologia e interventi di elezione.

Nel periodo postoperatorio sono necessari dei protocolli e procedure per il controllo del dolore, la prevenzione dei decubiti, la gestione del catetere vescicale, la funzione intestinale e la prevenzione dell'ileo paralitico, la somministrazione perioperatoria di ossigeno e l'idratazione, la prevenzione della malnutrizione, il controllo dell'anemia e la gestione delle trasfusioni.

Il paziente clinicamente compensato deve iniziare la mobilizzazione prima possibile ed essere successivamente trasferito in strutture protette per continuare il percorso riabilitativo sotto il controllo clinico di un team di specialisti di diverse discipline.

(G. Longo)

Le strategie prioritarie che devono essere adottate sono tutte le misure che devono portare alla stabilizzazione delle condizioni cliniche del paziente in modo da ridurre la mortalità postoperatoria a breve termine.

(A. Momoli)

Nella nostra esperienza l'utilizzo di vie chirurgiche minimamente invasive, di tecniche anestesiologiche sempre più selettive e di tecniche algologiche che permettano di avere un paziente collaborante ma senza dolore hanno sicuramente migliorato l'outcome dei pazienti protesizzati. Sempre più spesso nei nostri ambulatori si presentano pazienti giovani con richieste funzionali elevate che sono, a nostro modo di vedere, i pazienti più idonei a questo tipo di trattamento. La priorità va sempre di pari passo con i percorsi sicuri. L'utilizzo di anestesie selettive, di acido tranexamico e l'eliminazione dei drenaggi e del catetere vescicali ha sicuramente ridotto i rischi e migliorato l'outcome dei pazienti che possono riprendere la mobilizzazione in brevissimo tempo.

(F. Caroli)

La letteratura è coesa in tal senso: prioritario è operare il prima possibile questi pazienti. A tal fine, a mio avviso, la prima cosa da fare è educare tutti gli attori in gioco. Non parlo solo degli ortopedici, forse gli unici a rendersi veramente conto di questa necessità. Parlo delle direzioni politiche, amministrative e mediche degli ospedali e dei consulenti medici necessari allo scopo. Si tratta di medici di Pronto Soccorso, internisti, neurologi, geriatri, cardiologi e anestesisti che non di rado, anche se in buona fede, rallentano il processo terapeutico per "stabilizzare il paziente" e operarlo quando le "condizioni saranno migliorate" e le "comorbidità accertate". Bisogna inculcare loro che l'attesa è foriera di un aumento della mortalità. Devono considerare questi pazienti una vera urgenza, differibile, ma di molto poco.

(F. Randelli)

In relazione all'aumento delle aspettative di vita della popolazione occorre creare delle unità ortopediche geriatriche 
in cui il chirurgo ortopedico e il geriatra lavorino insieme con lo svolgimento di turni comuni, con anche la necessità di una riabilitazione immediata finalizzata al reinserimento del paziente nel contesto sociale e familiare antecedente al trauma.

(P. De Simoni)

\section{Pare utile implementare sistemi classificativi atti a stratificare pazienti portatori di rischio differenziato (paziente a basso rischio / a rischio contenuto / a maggior rischio) per perseguire l'appropriatezza della scelta terapeutica secondo un algoritmo che ne interpreta la complessità, favorendone l'analisi dei risultati secondo un criterio scientifico?}

Ritengo utile adottare sistemi classificativi validi per un'accurata analisi dei risultati che non possono prescindere dallo stato di salute dei pazienti.

(A. Campi)

Assolutamente Sİ.

(V. Caiaffa)

I rischi correlati al Fast-Track, nella breve degenza ospedaliera, hanno portato allo sviluppo di raccomandazioni di riferimento per la stratificazione del rischio e, conseguentemente, la gradazione degli interventi con l'obiettivo di una valida scelta terapeutica.

Il rischio stima la probabilità che si verifichi l'evento lesivo nell'intervento; può essere dipendente dal paziente e/o dall'esecuzione della tecnica chirurgica, in genere prevedibile ma a volte non completamente prevenibile, in alcuni casi non determinabile non certezza in assenza dell'identificazione dei fattori ad esso correlabili.

L'aumento del rischio è legato alla coesistenza di malattie associate (respiratorie, cardiovascolari, renali e cerebrali) e il disturbo cognitivo è uno degli aspetti dell'invecchiamento che suscita maggiore preoccupazione. Tuttavia, a volte, non vi è correlazione fra il timing chirurgico e la prognosi. Nell'identificazione dei fattori di rischio e nell'attribuzione della stratificazione differenziata, risulta evidente che il rischio è legato

(a) al quadro clinico

(b) alle caratteristiche dell'intervento chirurgico

(c) a indicazioni specifiche di disfunzioni organiche quali la funzionalità renale, una patologia ostruttiva bronchiale e il rischio coronarico. Inoltre, l'American Society of Anesthesiology (ASA) Score riassume la condizione globale del paziente nei confronti della procedura chirurgica-anestesiologica nel suo complesso.

L'obiettivo primario per l'appropriatezza delle scelte terapeutiche è di offrire all'operando un approccio multidisciplinare, dal ricovero alla dimissione. Dovranno essere implementate procedure evidence-based come trombo-profilassi, profilassi antibiotica, prevenzione delle ulcere da pressione, valutazione dello stato nutrizionale e standardizzazione del controllo del dolore.

La valutazione del rischio nell'operando deriva dal rischio individuale (dato dalle comorbidità dello stato anteriore al quadro clinico attuale), più quello attribuibile alla tipologia della frattura e dell'intervento. La stratificazione del rischio clinico permette di individuare i soggetti con quadro clinico a basso rischio, a rischio contenuto e a maggior rischio. I fattori legati al rischio chirurgico sono: la tipologia della frattura, le caratteristiche dell'intervento, il timing dell'intervento e la postura dell'operando sul lettino operatorio. L'identificazione e la gradazione del rischio chirurgico sono date dalla procedura anestesiologica.

In conclusione, si ritiene che la classificazione del rischio permetta all'operatore la scelta terapeutica più idonea, valutando il rischio/beneficio.

(F. Donelli)

A nostro giudizio è assolutamente necessario stratificare il rischio del paziente per costruire l'adeguato percorso di cura per singolo soggetto.

In particolare, la valutazione dei parametri cardiologici e anestesiologici prima di un eventuale ricovero in reparto di ortopedia renderebbe più sicura la gestione del malato.

(A. Momoli)

Riteniamo che vada stratificata nella valutazione preoperatoria una classificazione sul rischio al trattamento proposto operatorio e intraoperatorio dei vari pazienti. Inutile, a nostro giudizio, insistere con la mobilizzazione precocissima nel grande anziano e con la riabilitazione domiciliare e le tecniche di dimissione precoce, che portano poi il paziente ad essere parcheggiato in strutture che non hanno un knowhow sufficiente alla corretta gestione del protesizzato recente. Spesso il paziente anziano ha necessità di tempi di gestione più lunghi per imparare a riprendere uno schema motorio corretto. Diventa quindi imperativo classificare ex ante un rischio differenziato in cui vengano compresi fattori fisici, mentali e sociali. La stratificazione di un sistema classificativo alla prima valutazione fa partire un percorso più o meno virtuoso che inquadra il paziente lungo tutto il trattamento, consentendo alle figure professionali che dovranno gestirlo un protocollo standardizzato.

(F. Caroli)

Sistemi classificativi? Certamente sì, in futuro, e facendo studi prospettici e randomizzati. Ad oggi non c'è rischio che tenga. Vanno operati appena possibile e nella miglior sicurezza possibile, al momento. Si tratta di pazienti, spesso già in disequilibrio e a rischio imminente di vita, che in poche ore sviluppano un ulteriore decadimento. Quindi stratificare, senza una solida base scientifica, può voler dire incertezza e, quindi, perdita di tempo. Certo l'intervento non è una panacea ed è da considerare che esiste un'importante mortalità 
perioperatoria. I pazienti e i loro familiari devono esserne ben edotti. L'attesa non la modifica.

(F. Randelli)

Più che implementare sistemi classificativi è necessaria un'équipe multidisciplinare che segua il paziente in tutti i suoi aspetti, dalle comorbidità alla frattura; in particolare, in relazione all'aumentato impiego di farmaci anticoagulanti, occorre creare un algoritmo/linea guida per il corretto timing chirurgico in rapporto allo stato coagulativo del paziente. (P. De Simoni)

\section{Il Fast-Track rappresenta un mero obiettivo aziendale e un'obbligazione, ovvero costituisce un'efficace azione migliorativa che interpreta e corrisponde alle necessità del paziente?}

Il Fast-Track per le fratture del collo del femore dell'anziano è certamente un obiettivo aziendale ma è indubbio che, in quanto tale, abbia stimolato l'accelerazione nei tempi chirurgici con sostanziale beneficio per il paziente. Ma il Fast-Track deve esserci anche nel trattamento postoperatorio; non essendo obiettivo aziendale non viene sempre messo in atto, inficiando i benefici della ridotta tempistica preoperatoria. (A. Campi)

Non appare, a mio avviso, un mero obiettivo aziendale ma non può certamente essere il solo, se non l'unico, parametro di valutazione dell'attività di un'Unità Operativa di Ortopedia e Traumatologia, escludendo tutto quanto viene eseguito nella quotidiana attività lavorativa.

(B. Di Maggio)

Rappresenta uno dei rari casi in cui un obiettivo economico si sposa con un miglioramento della prestazione clinica.

(V. Caiaffa)

A seguito del Decreto 132 del 31/12/2013 della Regione Campania che approvava un Documento di "Indirizzo per la definizione del percorso assistenziale nelle fratture di femore nel paziente anziano", individuando "quale pratica cinica prioritaria l'intervento entro le 48 ore dall'accesso in P.S. del paziente anziano con diagnosi di frattura del collo del femore", di concerto con la Direzione Sanitaria e con le U.O.C. di Anestesia e Rianimazione e di Cardiologia del P.O. "S.G. Moscati" di Aversa abbiamo stilato e approvato un documento per il Fast-Track delle fratture del femore prossimale, nei pazienti over e under 65, riservando loro una sorta di corsia preferenziale già in P.S. con l'attribuzione al triage di un codice giallo e attivando un protocollo diagnostico-terapeutico con criteri di inclusione e esclusione all'intervento chirurgico nelle 48 ore.

A fronte del solo 12\% nel 2013, dei pazienti over 65 sono stati trattati entro le 48 ore dall'accesso in P.S. il 61,5\% nel 2014 (104 su 169), circa il 60\% nel 2015 (114 su 190) e il 67\% nel 2016 (125 su 186)—Dati Agenas P.N.E.: nel triennio 2014-
2016, in definitiva, le fratture del femore prossimale nei pazienti over 65 anni trattate chirurgicamente entro le 48 ore dall'accesso in P.S. sono state complessivamente 343 su 545 (63\%). Nel 2017 al 30/09 su 168 pazienti 125 (74,5\%) sono stati stabilizzati nelle $48 \mathrm{~h}$-Dati Archivio A.S.L.CE. L'introduzione del Fast-Track ha determinato una diminuzione sia della degenza media preoperatoria (3,4 giorni nel 2014, 3,2 nel 2015 e 2,6 nel 2016), sia postoperatoria (5,5 giorni nel $2014,4,5$ giorni nel 2015, 4 giorni nel 2016), con una marcata riduzione delle complicanze da "allettamento" quali ulcere e/o piaghe da decubito, infezioni e complicanze cardiorespiratorie e cardiocircolatorie nel preoperatorio e un'abolizione o marcata riduzione del dolore con conseguente riabilitazione precoce nel postoperatorio, così da evitare lunghe degenze ospedaliere e riabilitative e, quindi, un aggravio di costi diretti, indiretti e anche sociali.

Ciò nonostante, a nostro parere, pazienti affetti da comorbidità rilevanti e necessarie da essere inquadrate dal punto di vista clinico-strumentale e immediatamente corrette prima dell'intervento chirurgico, non sempre debbono essere trattati entro le 48 ore: riteniamo infatti che, in questi casi, l'intervento chirurgico debba essere eseguito quando sia stata ottenuta una stabilizzazione e una correzione di tutte le criticità organiche avvalendosi di un costante monitoraggio generale, onde evitare complicanze, talora anche letali, con susseguenti risvolti medico-legali.

Per i motivi sopra descritti riteniamo che il Fast-Track non rappresenti assolutamente un mero obiettivo aziendale ma un'azione efficace e tempestiva tale che il "paziente fragile", sia "anziano" che "grande anziano", possa beneficiare di cure che assicurino il ritorno a compiere gli atti quotidiani della vita quo ante l'evento traumatico e una minore incidenza di complicanze e di aggravamenti di condizioni cliniche pregresse già compromesse.

(A. Pellegrino)

Il Fast-Track è un'efficace misura se il paziente da trattare risulta stabile nelle sue condizioni cliniche. L'importante è parametrare l'obiettivo aziendale in funzione della tipologia di Ospedale e U.O., dei carichi di lavori richiesti e dalla tipologia di pazienti da trattare.

(A. Momoli)

Se gestito secondo principi sanitari e non amministrativi, il Fast-Track, a mio avviso, è un valido strumento che ci porterà a una ridotta ospedalizzazione, al recupero di energie da profondere in altre attività, con una soddisfazione che vede come attore l'utente e come risultato un nuovo modo di gestire la malattia. L'ospedale come luogo della "riparazione" e i servizi territoriali come valido supporto alla riabilitazione e al reinserimento dell'utente nella comunità, permettendo una rapida ripresa dell'autonomia che consente una più completa integrazione tra i vari attori della sanità.

(F. Caroli) 
No, il Fast-Track è una necessità in questi pazienti. Però deve essere fatto anche con una valutazione delle risorse a disposizione e una razionalizzazione. Non si può pensare, con le risorse mediche a disposizione oggi, di operare alle tre di notte un paziente con frattura del collo del femore, magari in coda a interventi eseguiti in urgenza dai chirurghi generali. Gli ortopedici italiani sono ai limiti per quantità di lavoro rischiano il burnout. Non devono pagare loro lo scotto di una cattiva gestione organizzativa e delle risorse più spesso destinate a favore di altri.

(F. Randelli)

Il Fast-Track rappresenta solo l'inizio di un percorso la cui conclusione deve essere la riduzione della degenza e il recupero più precoce possibile dell'autonomia della persona, per cui è necessario che, oltre al periodo pre-intervento, sia ottimizzato anche il periodo post-intervento.

(P. De Simoni)

\section{Quali sono le proposte di evoluzione del modello} organizzativo per la sostenibilità dell'azione assistenziale? Le proposte, a mio parere, traendo spunto dalla mia realtà ospedaliera, sono quelle di proseguire sulla strada delle 24-48 ore. Per migliorare e centrare questo obiettivo "aziendale" è necessaria un'organizzazione migliore che ancor più crei dei percorsi preferenziali per questi pazienti; ma poiché ci sono altri obiettivi di importanza primaria, anche se non aziendali, quale il recupero rapido alla vita attiva, bisogna lavorare anche per un Fast-Track riabilitativo. Troppo spesso si centra l'obiettivo aziendale e si perde il paziente che per giorni viene lasciato a letto.

Non esistono, poi, solo le fratture del collo del femore e trascurare le altre per favorire queste può far anteporre gli obiettivi aziendali all'obiettivo umano di trattare tutti i traumi nei tempi ottimali.

(A. Campi)

Sono profondamente convinto che il Fast-Track inizi dall'accettazione in Pronto Soccorso e vada necessariamente eseguito celermente non soltanto nel reparto di Traumatologia, ma unitamente a tutti i consulenti che, clinicamente e strumentalmente, contribuiranno in maniera determinante al completamento del complesso e articolato percorso assistenziale preoperatorio che ci consente poi di sottoporre il paziente all'intervento chirurgico; per tali motivi, l'attivazione di un diagramma funzionale sul quale possa essere strutturato un PDTA che preveda un flusso di informazioni reciproco tra territorio / reparto di degenza / sala operatoria / reparto / territorio potrebbe apportare un significativo miglioramento del funzionamento del Fast-Track.

(B. Di Maggio)
Percorsi dedicati.

(V. Caiaffa)

L'evoluzione del modello organizzativo dovrebbe portare a ridurre le troppe differenza presenti nel territorio nazionale nella gestione del paziente.

Ortopedici, geriatri e fisiatri dovrebbero auspicabilmente creare un modello gestionale da applicare al percorso di cura dei pazienti in tutti, o quasi, gli ospedali.

(A Momoli)

A nostro avviso, il nodo centrale del Fast-Track e il prericovero, luogo dove il paziente riceve tutte le informazioni sul suo intervento, sulla proposta terapeutica e dove la struttura elabora le strategie post-chirurgiche. Il vissuto dell'utente, la sua vita sociale nel suo contesto familiare determinano l'outcome terapeutico. Al pre-ricovero si possono valutare le condizioni assistenziali familiari, i contatti territoriali e le possibilità che il paziente possa accedere in tempi brevi e in sicurezza a strutture intermedie per il grande anziano, piuttosto che a servizi riabilitativi territoriali per l'utente attivo. Spesso, e sempre più, i nostri pazienti sono persone ancora inserite in ambiti lavorativi e richiedono pertanto un percorso individualizzato riabilitativo. Tutto ciò si riflette sui costi sociali che le strutture ospedaliere si trovano a gestire. Una grossa scommessa che la Regione Lombardia sta iniziando ad affrontare con la nuova riforma 23/2015.

(F. Caroli)

Bisogna investire risorse economiche per avere spazi specifici e dedicati a questi pazienti. Auspicabili dei Centri dedicati che coprano un ampio territorio e che siano sempre pronti, con un team multidisciplinare, a coprire le particolari esigenze di questa epidemia di pazienti. Legati tra loro da una rete informativa dedicata del 118. Ortopedici, come leader del team, coadiuvati da internisti, cardiologi, geriatri, anestesisti, neurologi e personale infermieristico. Tutto con un solo scopo e tutti iper-specializzati a tal fine. Non bisogna dimenticare infatti che, per vari motivi, si tratta di interventi con un alto tasso di complicazioni. Specializzare un team, soprattutto chirurgico, vuol dire ridurre le complicazioni. Quindi la mortalità.

\section{(F. Randelli)}

Modello organizzativo ideale è quello che prevede una collaborazione tra il chirurgo ortopedico, l'anestesista, il geriatra e il fisiatra In modo tale da coprire tutto il percorso dal trauma alla riabilitazione.

(P. De Simoni) 